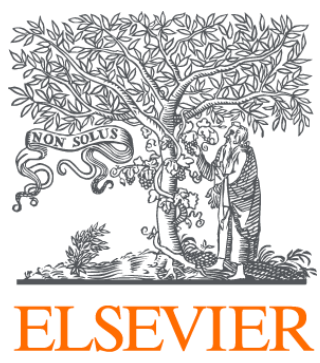

Since January 2020 Elsevier has created a COVID-19 resource centre with free information in English and Mandarin on the novel coronavirus COVID-

19. The COVID-19 resource centre is hosted on Elsevier Connect, the company's public news and information website.

Elsevier hereby grants permission to make all its COVID-19-related research that is available on the COVID-19 resource centre - including this research content - immediately available in PubMed Central and other publicly funded repositories, such as the WHO COVID database with rights for unrestricted research re-use and analyses in any form or by any means with acknowledgement of the original source. These permissions are granted for free by Elsevier for as long as the COVID-19 resource centre remains active. 


\section{European Journal of Internal Medicine}

Letter to the Editor

\section{Hospital preparedness for knowledge-based response to Ebola and other emerging infectious diseases: A continuous challenge}

The comment from Wiwanitkit stimulates some interesting remarks. Indeed, we agree that a "preparedness for knowledge" is essential for effective actions, especially in the case of an emerging disease such as Ebola, about which many gaps in knowledge still persist [1]. We also agree that, despite the relevant efforts and investments in resource allocation that have characterized the past two decades, adequate public health and clinical response to emerging diseases remain very challenging.

These gaps in knowledge already caused serious consequences in several occasions, especially regarding emerging and unusual disease. A report highlights that malaria misdiagnosis is frequent in western countries, and errors in malaria treatments occur in $25 \%$ of cases in the United States [2]; during the outbreak of West Nile Virus in New York City in 1999, the first case occurred 14 days before the first report to public health authorities by a clever physician who managed 2 unusual cases of encephalitis and 20 days before the starting of the epidemiological investigation [3]; during 2009 Influenza A(H1N1) pandemic many life-threatening diseases were erroneously labeled as swine flu [4,5]; and more recently have been reported many mistakes occurred during the management of the Liberian man affected by Ebola at Texas Health Presbyterian Hospital in Dallas, who was initially sent at home after his first visit to the Emergency Department [6].

As clearly stated by D.A. Henderson about 15 years ago "the involvement and expertise of infectious disease physicians, microbiologists, and public health practitioners are essential to the early detection and management of epidemics" [3].

These gaps in knowledge occur every time that a disease emerge or re-emerge, and should be filled as soon as possible, since the western countries are currently facing new challenging threats. Climate change offers new environmental niches for pathogens and vectors, while the increased global interconnectedness enhances the risk of importation of severe emerging diseases, as recently occurred for Ebola and Middle-East Respiratory Syndrome caused by a novel human coronavirus. In the meanwhile, Multi Drug Resistant Organisms continues to be reported in nosocomial settings, West Nile Virus and Crimean-Congo Haemorrhagic Fever virus are spreading in new geographic areas and cases of autochthonous malaria in new areas are described. In order to comply with these new challenges, preparedness activities are continuously needed, and these actions should rely, as properly suggested by Prof. Wiwanitkit, on development of new diagnostic tools, organization of multidisciplinary international collaborations, and establishment of effective isolation measures. The response to SARS epidemic was a clear example of how a networked health system can respond to a new unknown infectious threat to human health. The causative agent was identified and a reliable diagnostic was developed, thanks to a strong international collaboration, and isolation measures were strictly applied. These measures allowed the rapid control of the outbreak in the few subsequent months [7].

In this regard, an experience of "knowledge building" action is represented by the ETIDE (European Training for Infectious Diseases Emergencies) project, a network co-financed by the European Commission and managed by our Institute [8]. Under the umbrella of this project, front-line healthcare professionals from almost all European countries underwent to an integrated multidisciplinary training program about emerging, re-emerging and highly infectious diseases, in order to create a network of trained specialists which will facilitate communication and collaboration among European countries.

On the other hand, we believe that essential actions for hospital preparedness could be performed even in absence of a detailed knowledge about the next threat to face. A surveillance approach based on syndromes, the scrupulous application of standard and transmissionbased precautions, the accessibility to advanced diagnostic expertise and resources, the availability of adequate infrastructures and logistics for effective isolation, the constitution of strong international links and networks, all these actions could be undertaken independently by the next disease that will emerge. These preparedness measures do not have an "optimal duration", as Prof. Wiwanitkit asks for, but should represent a living continuous process, at least in leading institutions dedicated to the research on and the care of infectious diseases.

This long-lasting vision guided our steps in these 20 years of preparedness to emerging infectious diseases in our Institute.

\section{Conflict of interest}

All of the authors declare no conflict of interest.

\section{Acknowledgments}

We acknowledge the other members of INMI Preparedness and Response Groupe: Simone Lanini, Francesco Nicola Lauria, Silvia Meschi, Emanuele Nicastri, Carla Nisii, Nicola Petrosillo, Silvia Pittalis, Damiano Travaglini, and Francesco Vairo.

\section{References}

[1] Wiwanitkit V. Ebola virus infection: what should be known? N Am J Med Sci Nov 2014;6(11):549-52.

[2] Singh K, Wester WC, Trenholme GM. Problems in the therapy for imported malaria in the United States. Arch Intern Med Sep 22 2003;163(17):2027-30.

[3] Henderson DA, Inglesby Jr TV, O'Toole T, Fine A, Layton M. Lessons from the West Nile viral encephalitis outbreak in New York City, 1999: implications for bioterrorism preparedness. Clin Infect Dis 2001;32(2):277-82.

[4] Houlihan CF, Patel S, Price DA, Valappil M, Schwab U. A/H1N1 flu pandemic Life threatening infections labelled swine flu. BMJ Jan 13 2010;340:c137. http://dx.doi. org/10.1136/bmj.c137.

[5] Gunson RN, Carman WF. During the summer 2009 outbreak of "swine flu" in Scotland what respiratory pathogens were diagnosed as H1N1/2009? BMC Infect Dis Jul 13 2011;11:192. http://dx.doi.org/10.1186/1471-2334-11-192.

[6] Everything that went wrong in Dallas. Available at: Available at: http://www.slate. com/articles/health_and_science/medical_examiner/2014/10/dallas_ebola_timeline the_many_medical_missteps_at_texas_health_presbyterian.html. 
[7] Khabbaz RF. Still learning from SARS. Ann Intern Med Dec 3 2013;159(11):780-1.

[8] Bannister B, Prygodzicz A, Ippolito G, ETIDE Working Group. Training health care workers to face highly infectious diseases. Clin Microbiol Infect Aug 2009;15(8): $740-2$.

\section{Antonino Di Caro}

Vincenzo Puro

Francesco M. Fusco

Maria Rosaria Capobianchi

Giuseppe Ippolito*

on behalf of INMI Preparedness and Response Group

National Institute for Infectious Diseases (INMI) “L. Spallanzani”,

Rome, Italy

*Corresponding author at: National Institute for Infectious Diseases

“Lazzaro Spallanzani”, Via Portuense 292, 00149, Rome, Italy.

Tel.: + 390655170700 .

E-mail address: (G. Ippolito). 